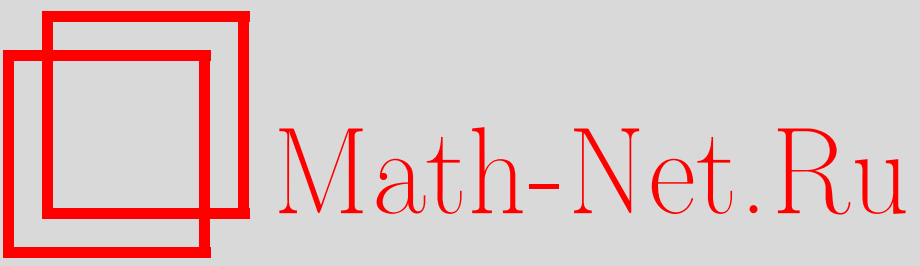

В. И. Денисов, Движение массивной частицы в окрестности сингулярной сферы, ТМФ, 1997, том 112, номер 2, 337-352

DOI: https://doi.org/10.4213/tmf1047

Использование Общероссийского математического портала Math-Net.Ru подразумевает, что вы прочитали и согласны с пользовательским соглашением

http://www.mathnet.ru/rus/agreement

Параметры загрузки:

IP: 54.197 .130 .99

26 апреля 2023 г., 13:00:39 


\section{ДВИЖЕНИЕ МАССИВНОЙ ЧАСТИЦЫ В ОКРЕСТНОСТИ СИНГУЛЯРНОЙ СФЕРЫ}

Проведено изучение движения массивной частицы в окрестности сингулярности статического сферически-симметричного решения уравнений релятивистской теории гравитации. Показано, что наличие массы у гравитона приводит к появлению качественно новых траекторий движения массивных частиц.

Как известно [1-2], приближенное решение уравнений релятивистской теории гравитации в случае статической сферически-симметричной метрики имеет вид

$$
\begin{gathered}
g_{00}=(1+2 m M) \frac{r-Z_{q}}{r}+q m^{2} M^{2}, \quad q>0, \\
g_{r r}=-\frac{r}{r-Z_{q}}, \quad g_{\theta \theta}=-r^{2}, \quad g_{\phi \phi}=-r^{2} \sin ^{2} \theta,
\end{gathered}
$$

где $m$ - масса гравитона, $M$ - масса источника гравитационного поля, $Z_{q}=2 M-$ $\nu m^{2} M^{2} \ln m M$, причем $\nu>0$.

Так как безразмерный параметр $\xi=m M$, по степеням разложения которого строилось решение $(1)$, очень мал $\left(\xi \sim 10^{-21}\right.$ даже для звезд-гигантов), то выражение (1) должно хорошо описывать точное решение уравнений релятивистской теории гравитации в достаточно большой области пространства. Поэтому при дальнейших вычислениях мы и будем использовать $(1)$, полагая $\xi \ll 1$ и интересуясь движением частищ в области пространства $r \ll 10^{28}$ см.

Следует отметить, что поскольку используемая в выражении (1) переменная $x^{0}$ эффективного псевдориманова пространства-времени является координатной величиной, отражающей некоторый частный выбор арифметизашии точек временной оси, то мы можем, не ограничивая обшности, использовать и другие способы арифметизации точек этой оси.

Для наших целей удобнее перейти в выражениях (1) от переменной $x^{0}$ к переменной $x^{\prime}=x^{0} \sqrt{1+2 m M+q m^{2} M^{2}}$, увеличив "темп хода" координатных часов в $\sqrt{1+2 m M+q m^{2} M^{2}}$ раз. Как известно [3], такое преобразование не выводит нас из первоначальной системы отсчета и совершенно не сказьвается на физически наблюдаемых величинах, тем более что при вычислениях мы будем использовать не координатное, а собственное время массивной частицы.

${ }^{*}$ Московский государственный университет, Москва, Россия 
Переходя в выражениях (1) к новому координатному времени и опуская в полученных соотношениях штрихи, будем иметь

$$
\begin{gathered}
g_{00}=U(r)=1-\frac{B Z_{q}}{r}, \\
g_{r r}=-V(r)=-\frac{r}{r-Z_{q}}, \quad g_{\theta \theta}=-r^{2}, \quad g_{\phi \phi}=-r^{2} \sin ^{2} \theta,
\end{gathered}
$$

где $B=(1+2 m M) /\left(1+2 m M+q m^{2} M^{2}\right)<1$.

Изучим движение массивных частиц в гравитационном поле (2). Запишем уравнение геодезического движения

$$
\frac{d u^{i}}{d s}+\Gamma_{n l}^{i} u^{n} u^{l}=0 .
$$

Так как траектория движения массивной частицы в псевдоримановом пространстве-времени (2) лежит на плоскости, то выберем систему координат так, чтобы эта плоскость совпала с плоскостью $\theta=\pi / 2$. Тогда уравнение $(3)$ примет вид

$$
\begin{gathered}
\frac{d u^{0}}{d s}+\frac{U^{\prime}}{U} u^{0} u^{r}=0 \\
\frac{d u^{r}}{d s}+\frac{U^{\prime}}{2 V}\left(u^{0}\right)^{2}-\frac{r}{V}\left(u^{\phi}\right)^{2}+\frac{V^{\prime}}{2 V}\left(u^{r}\right)^{2}=0 \\
\frac{d u^{\phi}}{d s}+\frac{2}{r} u^{\phi} u^{r}=0
\end{gathered}
$$

где штрих обозначает производную по $r$.

Несложно убедиться, что первое и третье уравнения дают

$$
u^{0}=E_{0} / U, \quad u^{\phi}=\alpha / r^{2}
$$

где $E_{0}$ и $\alpha$ - константы интегрирования, зависящие от начальных значений $r(0), \dot{r}(0)$, $\phi(0)$ и $\dot{\phi}(0)$.

Подставляя выражения (5) в условие $u^{i} u_{i}=1$, можно найти первьй интеграл для второго уравнения системы (4):

$$
\left(u^{r}\right)^{2}=\frac{1}{V}\left\{\frac{E_{0}^{2}}{U}-\frac{\alpha^{2}}{r^{2}}-1\right\} .
$$

Уравнения (4)-(6) позволяют провести полное исследование законов движения массивных частиц в гравитационном поле (2).

Рассмотрим сначала радиальное движение. В этом случае $\alpha=0$ и уравнение (6) принимает вид

$$
\left(\frac{d r}{d \tau}\right)^{2}=\frac{\left(r-Z_{q}\right)\left[\left(E_{0}^{2}-1\right) r+B Z_{q}\right]}{r\left(r-B Z_{q}\right)}
$$

Так как квадрат радиальной скорости частицы должен быть неотрицательной величиной, то в области $r \geq Z_{q}$ в зависимости от величины константы интегрирования $E_{0}^{2}$ возможны следуюшие три случая. 
Если $E_{0}^{2}>1$, то массивная частица может уйти на пространственную бесконечность и иметь там ненулевую радиальную скорость, измеренную по собственному времени частицы: $v_{\infty}^{2}=\left(E_{0}^{2}-1\right) / E_{0}^{2}$.

Если $E_{0}^{2}=1$, то массивная частища также может уйти на пространственную бесконечность, но радиальная скорость ее при этом будет асимптотически стремиться к нулю.

И, наконец, если $E_{0}^{2}<1$, то максимальное расстояние от тяготеющего центра, на которое может удалиться радиально движушаяся массивная частица, равно $r_{\max }=$ $B Z_{q} /\left(1-E_{0}^{2}\right)$.

Так как $r_{\max }$ должно удовлетворять условию $r_{\max } \geq Z_{q}$, то величина константы интегрирования $E_{0}^{2}$ для радиально движушейся массивной частицы ограничена снизу: $E_{0}^{2} \geq 1-B$.

Используя второе уравнение системы (4), несложно убедиться, что радиальное ускорение массивной частицы, измеренное по ее собственному времени, будет иметь вид

$$
\frac{d^{2} r}{d \tau^{2}}=\frac{Z_{q}}{2 r^{2}\left(r-B Z_{q}\right)^{2}}\left\{\left[E_{0}^{2}-1-B E_{0}^{2}\right] r^{2}+2 B Z_{q} r-B^{2} Z_{q}^{2}\right\} .
$$

Из этого выражения следует, что при $E_{0}^{2}<1 /(1-B)$ ускорение частицы будет отрицательно в области $r^{*}<r<\infty$ и положительно в области $Z_{q}<r<r^{*}$, где

$$
r^{*}=\frac{B Z_{q}}{1-\sqrt{(1-B) E_{0}^{2}}} .
$$

Это означает, что при радиальном движении в первой из указанных областей на частищу действует сила притяжения к источнику гравитационного поля, а во второй области сила отталкивания. На границе этих областей радиальная сила обращается в нуль.

При $E_{0}^{2} \geq 1 /(1-B)$ (т.е. для частиц ультравысоких энергий) сфера действия сил отталкивания расширяется на всю область радиального движения частищы $Z_{q}<r<\infty$.

Как следует из выражения (9), радиус $r^{*}$ существенно зависит от константы интегрирования $E_{0}$, определяемой начальной скоростью частицы, и от постоянных $B$ и $Z_{q}$, определяемых массой гравитона $m$ и массой источника тяготения $M$. Однако появление области отталкивания для частицы целиком обусловлено неравенством нулю массы гравитона. Если в выражении (8) совершить переход к общей теории относительности, положив $m=0$, то $B=1$, и мы получим $d^{2} r / d \tau^{2}=-Z_{q} /\left(2 r^{2}\right)<0$. Таким образом, в обшей теории относительности на радиально движушуюся частицу действует сила притяжения независимо от величины константы $E_{0}$.

В релятивистской теории гравитации область гравитационного отталкивания существует всегда, и измерение величины радиуса этой области по аккреции частиц и их электромагнитному излучению при известных энергии частицы и массе источника тяготения позволяет определить из наблюдаемых данных величину массы гравитона. Так как масса гравитона должна быть очень мала, то в первом порядке по $m$ из соотношения (9) можно получить следующее выражение для массы гравитона как функции параметров $E_{0}, Z_{q}$ и $M$, которые могут быть измерены: $m=\left(1-Z_{q} / r^{*}\right) /\left(\sqrt{q} M E_{0}\right)$.

Как показывают оценки, размер области гравитационного отталкивания $r^{*}$ значительно превышает гравитационный радиус $Z_{q}$ источника тяготения, если энергия налетаюшей частицы в $(m M)^{-1}$ раз превышает ее энергию покоя. Таким образом, если принять [4], что масса гравитона заключена в пределах $10^{-66}-10^{-65}$ г, то при массе источника, сравнимой с массой Солнца, радиус сферы гравитационного отталкивания будет 
значительно превышать гравитационньй радиус источника для ультрарелятивистских частиц, энергия которых в $10^{23}$ раз превьпает их энергию покоя.

Интегрируя уравнение (7), приходим к следуюшему закону радиального движения $\tau=\tau(r)$ массивной частицы в гравитационном поле $(2)$ :

$$
\tau= \pm \int_{r_{0}}^{r} d r \sqrt{\frac{r\left(r-B Z_{q}\right)}{\left(r-Z_{q}\right)\left[\left(E_{0}^{2}-1\right) r+B Z_{q}\right]}}
$$

где $r_{0}$ - радиальная координата частицы в начальньй момент собственного времени частицы $\tau=0$, знак плюс соответствует движению от тяготеющего центра, а знак минуск центру.

Этот интеграл может быть представлен в виде суммы элементарных и эллиптических функций.

При $m=0$ выражение (10) переходит в соответствующее решение общей теории относительности.

Так как при $E_{0}^{2} \geq 1$ в области $r \geq Z_{q}$ подынтегральное выражение имеет особенность вида $\left(r-Z_{q}\right)^{-1 / 2}$, то очевидно, что точка $r=Z_{q}$ будет точкой поворота для движущейся частищы.

Действительно, подставляя $r=Z_{q}$ в соотношения (7) и (8), несложно убедиться, что в этой точке радиальная скорость частицы равна нулю, а радиальное ускорение положительно. Поэтому после достижения сферы $r=Z_{q}$ массивная частица начнет движение в обратном направлении и при $E_{0}^{2} \geq 1$ уйдет на пространственную бесконечность (инфинитное движение).

Как следует из выражения (10), массивная частица при движении к центру из точки $r_{0}<\infty$ достигает сингулярную точку $r=Z_{q}$ за конечное собственное время:

$$
\tau=\int_{Z_{q}}^{r_{0}} d r \sqrt{\frac{r\left(r-B Z_{q}\right)}{\left(r-Z_{q}\right)\left[\left(E_{0}^{2}-1\right) r+B Z_{q}\right]}}
$$

Время этого движения остается конечным и при измерении по часам любого наблюдателя в силу того, что при $r \geq Z_{q}$ функция $U(r)$ нигде не обрашается в нуль.

Если же $E_{0}^{2}<1$, то аналогично можно доказать, что частица будет совершать периодические (но не гармонические) колебания от точки $r=Z_{q}$ до точки $r=r_{\max }$. Период колебаний частищы, измеренный по ее собственному времени, будет определяться выражением

$$
T_{2}=2 \int_{Z_{q}}^{r_{\max }} d r \sqrt{\frac{r\left(r-B Z_{q}\right)}{\left(r-Z_{q}\right)\left[\left(E_{0}^{2}-1\right) r+B Z_{q}\right]}} .
$$

Период этих же колебаний по координатному времени, совпадающему с собственным временем наблюдателя, находяшегося на бесконечности, будет равен

$$
T_{1}=2 E_{0} \int_{Z_{q}}^{r_{\max }} \frac{\sqrt{r^{3}} d r}{\sqrt{\left(r-B Z_{q}\right)\left(r-Z_{q}\right)\left[\left(E_{0}^{2}-1\right) r+B Z_{q}\right]}}
$$


Рассмотрим теперь нерадиальное движение массивной частицы. Для этого, используя второе из соотношений (5), удобно перейти в выражении (7) от производной по времени к производной по полярному углу $\phi$ :

$$
\left(\frac{d r}{d \phi}\right)^{2}=\frac{r\left(r-Z_{q}\right)\left[\left(E_{0}^{2}-1\right) r^{3}+B Z_{q} r^{2}-\alpha^{2} r+\alpha^{2} B Z_{q}\right]}{\alpha^{2}\left(r-B Z_{q}\right)}
$$

Совершенно аналогично второе уравнение системы (4) с учетом соотношений (5) приведем к виду, удобному для дальнейшего исследования,

$$
\begin{aligned}
\frac{d^{2} r}{d \phi^{2}}= & \frac{\left(4 r-3 Z_{q}\right)\left[\left(E_{0}^{2}-1\right) r^{3}+B Z_{q} r^{2}-\alpha^{2} r+\alpha^{2} B Z_{q}\right]}{2 \alpha^{2}\left(r-B Z_{q}\right)}+ \\
& +\left(r-Z_{q}\right)\left[1-\frac{E_{0}^{2} B Z_{q} r^{3}}{2 \alpha^{2}\left(r-B Z_{q}\right)^{2}}\right]
\end{aligned}
$$

Вид траектории полностью определяется поведением функции, стоящей в правой части равенства (11), а точнее, взаимным расположением и кратностью нулей этой функции.

Так как движение массивной частицы изучается нами в области $r \geq Z_{q}$, а $B<1$, то корни функции $(d r / d \phi)^{2}$ в этой области совпадают с корнями уравнения

$$
f(r)=\left(E_{0}^{2}-1\right) r^{3}+B Z_{q} r^{2}-\alpha^{2} r+\alpha^{2} B Z_{q}=0
$$

и, кроме того, один корень лежит на сфере $r=Z_{q}$. Уравнение же (13) может иметь от одного до трех действительных корней в зависимости от соотношения между величинами $E_{0}, \alpha$, характеризующими начальные условия для массивной частицы, и величинами $B, Z_{q}$, зависящими от массы $M$ источника тяготения и массы $m$ гравитона.

Рассмотрим все случаи, которые могут быть реализованы различным выбором начальных условий для движушейся частицы. Начнем с самого простого для анализа частного значения $E_{0}^{2}=1$.

В этом случае уравнение (13) превращается в квадратное, дискриминант которого $d=\alpha^{2}-4 B^{2} Z_{q}^{2}$. Поэтому данное уравнение будет иметь два различных положительных вешественных корня

$$
r_{1,2}=\frac{\alpha}{2 B Z_{q}}\left[\alpha \pm \sqrt{\alpha^{2}-4 B^{2} Z_{q}^{2}}\right]
$$

при $\alpha^{2}>4 B^{2} Z_{q}^{2}$, кратные корни $r_{1}=r_{2}=2 B Z_{q}$ при $\alpha^{2}=4 B^{2} Z_{q}^{2}$ и комплексные корни

$$
r_{1,2}=\frac{\alpha}{2 B Z_{q}}\left[\alpha \pm i \sqrt{4 B^{2} Z_{q}^{2}-\alpha^{2}}\right]
$$

при $\alpha^{2}<4 B^{2} Z_{q}^{2}$.

Изучим траектории движения массивной частицы, которые возможны во всех перечисленных случаях. Полагая в уравнении (11) $E_{0}^{2}=1$ и интегрируя его, получим

$$
\phi=\phi_{0} \pm \frac{\alpha}{\sqrt{B Z_{q}}} \int_{r_{0}}^{r} d r \sqrt{\frac{\left(r-B Z_{q}\right)}{r\left(r-Z_{q}\right)\left(r-r_{1}\right)\left(r-r_{2}\right)}},
$$


где $r_{0}$ и $\phi_{0}-$ полярные координаты частицы в начальный момент собственного времени $\tau=0$ и знак перед интегралом должен быть выбран в соответствии со знаком $d r / d \phi$ при $\tau=0$. В дальнейшем для определенности мы будем полагать, что $\alpha d r / d \phi>0$.

Предположим, что в силу начальных условий выполняется соотношение $\alpha^{2}>$ $4 B^{2} Z_{q}^{2}$. Тогда в зависимости от величины постоянных $\alpha^{2}, B$ и $Z_{q}$ вешественные и положительные корни $r_{1}$ и $r_{2}$ могут иметь различное расположение относительно точки $r=Z_{q}$.

Рассмотрим по очереди возникаюшие здесь случаи.

1. Пусть $E_{0}^{2}=1$ и $Z_{q}<r_{2}<r_{1}$. Как следует из выражения (14), это условие будет выполнено, если $4 B^{2} Z_{q}^{2}<\alpha^{2}<B Z_{q}^{2} /(1-B)$. Здесь условие неотрицательности правой части уравнения (11) допускает движение частицы только в двух областях пространства: $Z_{q} \leq r \leq r_{2}$ и $r \geq r_{1}$. В каждой из этих областей имеется своя траектория частищы; двигаясь по траектории, лежащей в одной из этих областей, частица не в состоянии попасть в другую область. По какой из этих двух траекторий будет двигаться частица, полностью определяется начальным значением $r_{0}$.

1a. Изучим сначала траекторию, находящуюся в области $Z_{q}<r<r_{2}$. Так как подынтегральное выражение в уравнении траектории (16) имеет в области $r_{2} \geq r \geq Z_{q}$ особенность вида $\left[r-Z_{q}\right]^{-1 / 2}$ в точке $r=Z_{q}$ и особенность вида $\left[r-r_{2}\right]^{-1 / 2}$ в точке $r=r_{2}$, то из общей теории следует, что интеграл (16) принимает конечные значения при $r=Z_{q}$ и $r=r_{2}$. Поэтому траектория, проходящая через точку $Z_{q}<r_{0}<r_{2}$ и $\phi=\phi_{0}$, приближается к окружности $r=Z_{q}$ за конечное число оборотов, равное целому от

$$
\left|\frac{\phi-\phi_{0}}{2 \pi}\right|=\frac{|\alpha|}{2 \pi \sqrt{B Z_{q}}} \int_{Z_{q}}^{r_{0}} d r \sqrt{\frac{\left(r-B Z_{q}\right)}{r\left(r-Z_{q}\right)\left(r-r_{1}\right)\left(r-r_{2}\right)}}
$$

Так как в силу соотношения (12) $d^{2} r / d \phi^{2}>0$ в точке $r=Z_{q}$, то эта траектория, коснувшись окружности $r=Z_{q}$, при

$$
\phi=\phi_{m}^{*}=\phi_{0}+\frac{\alpha}{\sqrt{B Z_{q}}} \int_{Z_{q}}^{r_{0}} d r \sqrt{\frac{\left(r-B Z_{q}\right)}{r\left(r-Z_{q}\right)\left(r-r_{1}\right)\left(r-r_{2}\right)}}
$$

отражается от нее и начинает приближаться к окружности $r=r_{2}$. Ветви траектории $\phi<\phi_{m}^{*}$ и $\phi>\phi_{m}^{*}$ являются взаимно симметричными относительно полупрямой $\phi=\phi_{m}^{*}$ и отличаются лишь знаком производной $d r / d \phi$.

Достигнув окружности $r=r_{2}$ также за конечное число оборотов, траектория отражается от нее в силу того, что $d^{2} r / d \phi^{2}<0$ в точке $r=r_{2}$. Таким образом, в области $Z_{q} \leq r \leq r_{2}$ будет совершаться финитное движение.

Смешение перищентра частишы за один оборот при движении по такой траектории составляет

$$
\delta \phi=\frac{2 \alpha}{\sqrt{B Z_{q}}} \int_{Z_{q}}^{r_{2}} d r \sqrt{\frac{\left(r-B Z_{q}\right)}{r\left(r-Z_{q}\right)\left(r-r_{1}\right)\left(r-r_{2}\right)}}-2 \pi .
$$

1б. Пусть начальное положение частицы находится во второй области: $r_{0}>r_{1}$. Так как подынтегральное выражение равенства (16) при $r \rightarrow \infty$ убывает пропорционально $r^{-2}$, то при $r_{0}>r_{1}$ и $r \rightarrow \infty$ этот интеграл сходится. Это означает, что одна ветвь 
траектории уходит на пространственную бесконечность, совершив конечное число оборотов, и при $r \rightarrow \infty$ имеет асимптотой полупрямую $\phi=\phi_{\infty}$, где

$$
\phi_{\infty}=\phi_{0}+\frac{\alpha}{\sqrt{B Z_{q}}} \int_{r_{0}}^{\infty} d r \sqrt{\frac{\left(r-B Z_{q}\right)}{r\left(r-Z_{q}\right)\left(r-r_{1}\right)\left(r-r_{2}\right)}} .
$$

Другая ветвь траектории, проходящей через точку $r=r_{0}>r_{1}$ и $\phi=\phi_{0}$, приближается к окружности $r=r_{1}$, делая конечное число оборотов вокруг нее. Коснувшись этой окружности, траектория в силу условия $d^{2} r / d \phi^{2}>0$ при $r=r_{1}$ отражается от нее и, совершив конечное число оборотов вокруг тяготеюшего центра, уходит на пространственную бесконечность.

Таким образом, движение частишы в области $r \geq r_{1}$ будет инфинитным.

2. Пусть $E_{0}^{2}=1$ и $Z_{q}=r_{2}<r_{1}$. Как следует из выражения (14), это условие будет выполнено, если $\alpha^{2}=B Z_{q}^{2} /(1-B)$, причем $r_{1}=B Z_{q} /(1-B) \gg Z_{q}$.

В этом случае условие неотрицательности правой части уравнения (11) допускает движение частицы либо по окружности $r=Z_{q}$, либо в области пространства $r \geq r_{1}$ в зависимости от положения частицы в начальный момент времени.

Если $r_{0} \geq r_{1}$, то уравнение траектории (16) принимает вид

$$
\phi=\phi_{0}+\sqrt{\frac{Z_{q}}{1-B}} \int_{r_{0}}^{r} \frac{d r}{\left(r-Z_{q}\right)} \sqrt{\frac{\left(r-B Z_{q}\right)}{r\left(r-r_{1}\right)}} .
$$

Так как в области $r \geq r_{1}$ подынтегральное выражение имеет особенность вида $\left[r-r_{1}\right]^{-1 / 2}$ в точке $r=r_{1}$, то одна ветвь траектории, начинаясь в точке $r=r_{0}$, за конечное число оборотов приближается к окружности $r=r_{1}$, касается ее и из-за того, что $d^{2} r / d \phi^{2}>0$ при $r=r_{1}$, после касания уходит на пространственную бесконечность, совершив конечное число оборотов вокруг тяготеюшего центра.

Другая ветвь траектории, начинаясь в точке $r=r_{0}$, уходит на пространственную бесконечность, также совершив конечное число оборотов вокруг источника тяготения.

3. Пусть $E_{0}^{2}=1$ и $r_{2}<Z_{q}<r_{1}$. Как следует из выражения (14), это условие будет выполнено, если $\alpha^{2}>B Z_{q}^{2} /(1-B)$. В этом случае условие неотрицательности правой части уравнения (11) допускает движение частицы только в области $r \geq r_{1}$ и качественно поведение траектории в этой области не отличается от поведения траектории $r \geq r_{1}$, рассмотренной в п. 1б.

Используя выражения (14), можно показать, что при $\alpha^{2}>4 B^{2} Z_{q}^{2}$ выполняется условие $r_{1}>Z_{q}$, поэтому взаимное расположение вещественных и неравных корней $r_{1}, r_{2}$ относительно точки $r=Z_{q}$ исчерпывается указанными выше случаями.

4. Перейдем теперь к рассмотрению кратных корней уравнения (13) при $E_{0}^{2}=1$. В этом случае $\alpha= \pm 2 B Z_{q}$ и $r_{1}=r_{2}=2 B Z_{q}$, в результате чего условие неотрицательности правой части соотношения (11) допускает движение частищы во всей области пространства $Z_{q} \leq r<\infty$.

Используя явньй вид $\alpha, r_{1}$ и $r_{2}$, из выражения (16) получим

$$
\phi=\phi_{0} \pm 2 \sqrt{B Z_{q}} \int_{r_{0}}^{r} \frac{d r}{\left|r-2 B Z_{q}\right|} \sqrt{\frac{\left(r-B Z_{q}\right)}{r\left(r-Z_{q}\right)}} .
$$


Несложно убедиться, что этот интеграл расходится при $r \rightarrow 2 B Z_{q}$ и имеет конечные пределы при $r \rightarrow \infty$ и $r \rightarrow Z_{q}$. Это означает, что область пространства $r \geq Z_{q}$, в которой может происходить движение, разбивается на две подобласти $Z_{q} \leq r<2 B Z_{q}$ и $2 B Z_{q}<r<\infty$, в каждой из которых имеется своя траектория массивной частицы.

Если начальное положение частицы $r_{0}$ принадлежит первой области пространства, то траектория, проходяшая через точку $Z_{q}<r_{0}<2 B Z_{q}$ и $\phi=\phi_{0}$ приближается к окружности $r=Z_{q}$ за конечное число оборотов, в то время как к окружности $r=2 B Z_{q}$ траектория приближается асимптотически, закручиваясь по спирали бесконечное число раз. Так как в силу соотношения $(12) d^{2} r / d \phi^{2}>0$ в точке $r=Z_{q}$, то эта траектория, коснувшись окружности $r=Z_{q}$, при

$$
\phi=\phi_{m}=\phi_{0} \pm 2 \sqrt{B Z_{q}} \int_{r_{0}}^{Z_{q}} \frac{d r}{\left|r-2 B Z_{q}\right|} \sqrt{\frac{\left(r-B Z_{q}\right)}{r\left(r-Z_{q}\right)}}
$$

отражается от нее и начинает асимптотическое приближение к окружности $r=2 B Z_{q}$. Ветви траектории $\phi<\phi_{m}$ и $\phi>\phi_{m}$ являются взаимно симметричными и отличаются лишь знаком производной $d r / d \phi$.

Траектория, проходящая через точку $r_{0}>2 B Z_{q}$ и $\phi=\phi_{0}$, во второй области пространства также асимптотически приближается к окружности $r=2 B Z_{q}$, делая бесконечное число оборотов вокруг нее. Так как подынтегральное выражение равенства (16) при $r \rightarrow \infty$ убывает пропорционально $r^{-2}$, то при $r_{0}>2 B Z_{q}$ и $r \rightarrow \infty$ этот интеграл сходится.

Это означает, что другая ветвь этой траектории уходит на пространственную бесконечность, совершив конечное число оборотов, и при $r \rightarrow \infty$ имеет асимптотой полупрямую $\phi=\phi_{\infty}^{*}$, где

$$
\phi_{\infty}^{*}=\phi_{0} \pm 2 \sqrt{B Z_{q}} \int_{r_{0}}^{\infty} \frac{d r}{\left|r-2 B Z_{q}\right|} \sqrt{\frac{\left(r-B Z_{q}\right)}{r\left(r-Z_{q}\right)}}
$$

Поэтому если частища движется по этой траектории и в начальный момент собственного времени ее радиальная скорость положительна, то она по первой ветви траектории уйдет на пространственную бесконечность. Если же в начальный момент собственного времени радиальная скорость частицы отрицательна, то она будет бесконечно долго приближаться по спирали к окружности $r=2 B Z_{q}$. В этом случае можно говорить о гравитационном захвате частищы.

Если же выполняются условия $\alpha^{2}=4 B^{2} Z_{q}^{2}, E_{0}^{2}=1$ и начальное положение массивной частицы $r_{0}=2 B Z_{q}$, то, как следует из выражений (11) и (12), $d r / d \phi=d^{2} r / d \phi^{2}=0$. Это означает, что при $r_{0}=2 B Z_{q}$ частища будет двигаться по окружности радиуса $r=2 B Z_{q}$. Период ее обрашения по круговой орбите, измеренный по собственному времени, будет равен $T=4 \pi B Z_{q} / c$.

5. И, наконец, при выполнении условий $E_{0}^{2}=1$ и $\alpha^{2}<4 B^{2} Z_{q}^{2}$ выражение (16) примет вид

$$
\phi=\phi_{0}+2 \alpha \sqrt{B Z_{q}} \int_{r_{0}}^{r} d r \sqrt{\frac{\left(r-B Z_{q}\right)}{r\left(r-Z_{q}\right)\left[\left(2 B Z_{q} r-\alpha^{2}\right)^{2}+\alpha^{2}\left(4 B^{2} Z_{q}^{2}-\alpha^{2}\right)\right]}} .
$$


В этом случае условие неотрицательности правой части уравнения (11) допускает движение частицы во всей области $Z_{q} \leq r<\infty$. Несложный анализ, аналогичный проделанному выше, показывает, что в этой области пространства траектория качественно ведет себя так же, как траектория, описанная в п. 1б, за исключением того, что точкой поворота будет $r=Z_{q}$.

Рассмотрим теперь более сложную для анализа ситуацию, когда в силу начальных условий $E_{0}^{2}$ не равна единице.

Корни уравнения (13) в этом случае можно получить, используя известную формулу Кардано. Однако выражения для корней, найденные с использованием этой формулы, оказываются настолько сложными, что практически не позволяют проводить их анализ в обшем виде. Так как для наших целей важное значение имеет установление знаков вещественных корней уравнения (13), то воспользуемся теоремой Виета.

Обозначая корни функции (13) через $r_{1}, r_{2}$ и $r_{3}$, в силу теоремы Виета при $E_{0}^{2} \neq 1$ будем иметь

$$
\begin{gathered}
r_{1}+r_{2}+r_{3}=-B Z_{q} /\left(E_{0}^{2}-1\right), \\
r_{1} r_{2}+r_{1} r_{3}+r_{2} r_{3}=-\alpha^{2} /\left(E_{0}^{2}-1\right), \quad r_{1} r_{2} r_{3}=-\alpha^{2} B Z_{q} /\left(E_{0}^{2}-1\right) .
\end{gathered}
$$

Из этих уравнений следует, что знаки вешественных корней сушественно зависят от знака разности $E_{0}^{2}-1$. Если $E_{0}^{2} \geq 1$, то последнее из соотношений (17) означает, что один из вещественных корней (обозначим его через $r_{3}$ ) должен быть отрицательным. Два остальных корня могут быть либо комплексно-сопряженными, либо действительными (различными или одинаковыми).

Вычитая из третьего соотношения (17) первое, умноженное на второе, получим

$$
-\left[r_{1}+r_{2}\right]\left[r_{1} r_{2}+r_{3}\left(r_{1}+r_{2}+r_{3}\right)\right]=-\frac{\alpha^{2} E_{0}^{2} B Z_{q}}{\left(E_{0}^{2}-1\right)^{2}}<0 .
$$

Так как в этом случае $r_{1} r_{2}>0, r_{1}+r_{2}+r_{3}<0, r_{3}<0$, то отсюда следует, что и $r_{1}+r_{2}>0$. Это неравенство вместе с условием $r_{1} r_{2}>0$ означает, что если корни $r_{1}$ и $r_{2}$ при $E_{0}^{2} \geq 1$ вешественные, то они обязаны быть положительными.

Если $E_{0}^{2} \leq 1$, то последнее из соотношений (17) означает, что один из вешественных корней (обозначим его также через $r_{3}$ ) должен быть положительным. Так как в этом случае в силу соотношений (17) $r_{1} r_{2}>0, r_{1}+r_{2}+r_{3}>0, r_{3}>0$, то вычитая из третьего соотношения (17) первое, умноженное на второе, несложно показать, что и $r_{1}+r_{2}>0$. Поэтому при $E_{0}^{2} \leq 1$ вешественные корни $r_{1}$ и $r_{2}$ обязаны быть положительными.

Таким образом, знак $E_{0}^{2}-1$ сушественно влияет только на знак корня $r_{3}:$ при $E_{0}^{2}>1$ корень $r_{3}$ отрицательный, в то время как при $E_{0}^{2}<1$ он положительный. Если остальные два корня уравнения (13) являются вещественными, то они будут положительными независимо от знака $E_{0}^{2}-1$.

Число действительных корней уравнения (13) зависит от знака дискриминанта этого уравнения:

$$
D=\frac{\alpha^{2}\left[-4 \alpha^{4}\left(E_{0}^{2}-1\right)+\alpha^{2} B^{2} Z_{q}^{2}\left(27 E_{0}^{4}-36 E_{0}^{2}+8\right)+4 B^{4} Z_{q}^{4}\right]}{108\left(E_{0}^{2}-1\right)^{4}} .
$$

В частности, если $D>0$, то уравнение (13) имеет один действительный корень и два комплексных; при $D<0$ уравнение (13) имеет три действительных различных корня. 
И, наконец, при $D=0$ уравнение (13) имеет три действительных корня, среди которых имеются кратные.

Выясним, при каких значениях постоянной $E_{0}^{2}$ реализуются указанные вьше случаи. Так как $\alpha^{2} / 108\left(E_{0}^{2}-1\right)^{4}>0$, то знак дискриминанта $D$ совпадает со знаком функции

$$
F=-4 \alpha^{4}\left(E_{0}^{2}-1\right)+\alpha^{2} B^{2} Z_{q}^{2}\left(27 E_{0}^{4}-36 E_{0}^{2}+8\right)+4 B^{4} Z_{q}^{4} .
$$

Рассматривая эту функцию как квадратный трехчлен относительно $\alpha^{2}$, можно установить, как зависит знак дискриминанта $D$ кубического уравнения (13) от величины $E_{0}^{2}$. Для этого построим сначала дискриминант $d$ квадратного трехчлена (18). В результате получим

$$
d=b^{2}-4 a c=B^{4} Z_{q}^{4} E_{0}^{2}\left[9 E_{0}^{2}-8\right]^{3} .
$$

Используя это соотношение и выражение (18), мы можем классифицировать все возможные частные случаи в зависимости от величины параметра $E_{0}^{2}$ при условии, что $E_{0}^{2} \neq 1$. Если $E_{0}^{2}>1$, то дискриминант $d$ положителен. Поэтому квадратный трехчлен (18) (а следовательно, и дискриминант $D$ ) отрицателен при

$$
\alpha^{2}>\frac{B^{2} Z_{q}^{2}}{8\left(E_{0}^{2}-1\right)}\left[27 E_{0}^{4}-36 E_{0}^{2}+8+\sqrt{E_{0}^{2}\left(9 E_{0}^{2}-8\right)^{3}}\right],
$$

равен нулю при

$$
\alpha^{2}=\frac{B^{2} Z_{q}^{2}}{8\left(E_{0}^{2}-1\right)}\left[27 E_{0}^{4}-36 E_{0}^{2}+8+\sqrt{E_{0}^{2}\left(9 E_{0}^{2}-8\right)^{3}}\right]
$$

и положителен при $\alpha^{2}$, заключенном в пределах

$$
0<\alpha^{2}<\frac{B^{2} Z_{q}^{2}}{8\left(E_{0}^{2}-1\right)}\left[27 E_{0}^{4}-36 E_{0}^{2}+8+\sqrt{E_{0}^{2}\left(9 E_{0}^{2}-8\right)^{3}}\right]
$$

Это означает, что если $E_{0}^{2}>1$ и параметр $\alpha^{2}$ удовлетворяет условию (19), то уравнение (13) имеет три различных действительных корня (один отрицательный и два положительных); при выполнении соотношения (20) из трех действительных корней два корня (положительные) будут совпадать и при значении параметра $\alpha^{2}$, заключенном в пределах (21), уравнение (13) будет иметь один отрицательный действительный корень и два комплексно-сопряженных.

Совершенно аналогично можно показать, что при $8 / 9<E_{0}^{2}<1$ величина $\alpha^{2}$ также предопределяет тип корней уравнения (13).

В частности, вводя обозначения

$$
\begin{aligned}
& \alpha_{1}^{2}=\frac{B^{2} Z_{q}^{2}}{8\left(E_{0}^{2}-1\right)}\left[27 E_{0}^{4}-36 E_{0}^{2}+8+\sqrt{E_{0}^{2}\left(9 E_{0}^{2}-8\right)^{3}}\right], \\
& \alpha_{2}^{2}=\frac{B^{2} Z_{q}^{2}}{8\left(E_{0}^{2}-1\right)}\left[27 E_{0}^{4}-36 E_{0}^{2}+8-\sqrt{E_{0}^{2}\left(9 E_{0}^{2}-8\right)^{3}}\right]
\end{aligned}
$$

несложно убедиться, что если значение параметра $\alpha^{2}$ удовлетворяет неравенству

$$
\alpha_{1}^{2}<\alpha^{2}<\alpha_{2}^{2}
$$


то уравнение (13) будет иметь три различных действительных положительных корня; если выполняется одно из равенств

$$
\alpha^{2}=\alpha_{1}^{2} \text { или } \alpha^{2}=\alpha_{2}^{2},
$$

то из трех положительных корней два будут совпадать, при выполнении одного из неравенств

$$
0<\alpha^{2}<\alpha_{1}^{2} \text { или } \alpha^{2}>\alpha_{2}^{2}
$$

уравнение (13) будет иметь один положительный вешественный корень и два комплексно-сопряженных корня.

Если $E_{0}^{2}=8 / 9$, то дискриминант $D$ принимает вид $D=27 \alpha^{2}\left(\alpha^{2}-3 B^{2} Z_{q}^{2}\right)^{2}$. Отсюда непосредственно следует, что при $E_{0}^{2}=8 / 9$ и $\alpha^{2} \neq 3 B^{2} Z_{q}^{2}$ уравнение (13) имеет один положительный корень

$$
\begin{aligned}
r_{3}= & 3 B Z_{q}+\sqrt[3]{\left(\alpha^{2}-3 B^{2} Z_{q}^{2}\right)\left[\sqrt{27 \alpha^{2}}-9 B Z_{q}\right]}- \\
& -\sqrt[3]{\left(\alpha^{2}-3 B^{2} Z_{q}^{2}\right)\left[\sqrt{27 \alpha^{2}}+9 B Z_{q}\right]}
\end{aligned}
$$

и два комплексно-сопряженных.

Если же начальные условия обеспечивают выполнение равенств $E_{0}^{2}=8 / 9$ и $\alpha^{2}=$ $3 B^{2} Z_{q}^{2}$, то уравнение (13) будет иметь три совпадающих положительных корня: $r_{1}=$ $r_{2}=r_{3}=3 B Z_{q}$.

И, наконец, если $E_{0}^{2}<8 / 9$, то дискриминант $D$ будет положителен при любых значениях параметра $\alpha^{2}$, в результате чего уравнение (13) будет иметь только один положительный вещественный корень и два комплексно-сопряженных.

Рассмотрим последовательно все возможные случаи, возникаюшие при $E_{0}^{2} \neq 1$.

Пусть $E_{0}^{2}>1$ и выполняется условие (19). Интегрируя уравнение (11), в этом случае получим следуюшее выражение:

$$
\phi=\phi_{0}+\frac{\alpha}{\sqrt{E_{0}^{2}-1}} \int_{r_{0}}^{r} d r \sqrt{\frac{\left(r-B Z_{q}\right)}{r\left(r-Z_{q}\right)\left(r+a_{3}^{2}\right)\left(r-r_{2}\right)\left(r-r_{1}\right)}},
$$

где $r_{3}=-a_{3}^{2}, r_{2}, r_{1}$ - вешественные корни уравнения (13).

В зависимости от величины постоянных $E_{0}^{2}, \alpha^{2}, B$ и $Z_{q}$ корни $r_{1}$ и $r_{2}$ могут иметь различное расположение относительно точки $r=Z_{q}$.

6. Если $Z_{q}<r_{2}<r_{1}$, то условие неотрицательности правой части уравнения (11) допускает движение частицы только в двух областях пространства: $Z_{q} \leq r \leq r_{2}$ и $r \geq r_{1}$.

6а. Изучим сначала траекторию, находящуюся в области $Z_{q}<r<r_{2}$. Так как подынтегральное выражение в уравнении траектории (26) имеет в этой области особенность вида $\left[r-Z_{q}\right]^{-1 / 2}$ в точке $r=Z_{q}$ и особенность вида $\left[r-r_{2}\right]^{-1 / 2}$ в точке $r=r_{2}$, то из общей теории следует, что интеграл (26) принимает конечные значения при $r=Z_{q}$ и $r=r_{2}$. Поэтому траектория, проходящая через точку $Z_{q}<r_{0}<r_{2}$ и $\phi=\phi_{0}$, приближается к окружности $r=Z_{q}$ за конечное число оборотов, равное целому от

$$
\left|\frac{\phi-\phi_{0}}{2 \pi}\right|=\frac{\alpha}{2 \pi \sqrt{E_{0}^{2}-1}} \int_{Z_{q}}^{r_{0}} d r \sqrt{\frac{\left(r-B Z_{q}\right)}{r\left(r-Z_{q}\right)\left(r+a_{3}^{2}\right)\left(r-r_{2}\right)\left(r-r_{1}\right)}} .
$$


Так как в силу соотношения (12) $d^{2} r / d \phi^{2}>0$ в точке $r=Z_{q}$, то эта траектория, коснувшись окружности $r=Z_{q}$, при

$$
\phi=\phi_{0}+\frac{\alpha}{\sqrt{E_{0}^{2}-1}} \int_{r_{0}}^{Z_{q}} d r \sqrt{\frac{\left(r-B Z_{q}\right)}{r\left(r-Z_{q}\right)\left(r+a_{3}^{2}\right)\left(r-r_{2}\right)\left(r-r_{1}\right)}}
$$

отражается от нее и начинает приближаться к окружности $r=r_{2}$.

Достигнув окружности $r=r_{2}$ также за конечное число оборотов, траектория отражается от нее в силу того, что $d^{2} r / d \phi^{2}<0$ в точке $r=r_{1}$. Таким образом, в области $Z_{q} \leq r \leq r_{2}$ будет совершаться финитное движение.

6б. Траектория, проходяшая через точку $r_{0}>r_{1}$ и $\phi=\phi_{0}$, во второй области пространства также приближается к окружности $r=r_{1}$, делая конечное число оборотов вокруг нее. Так как подынтегральное выражение равенства (26) при $r \rightarrow \infty$ убывает пропорционально $r^{-2}$, то при $r_{0}>r_{1}$ и $r \rightarrow \infty$ этот интеграл сходится. Это означает, что другой конец этой траектории уходит на пространственную бесконечность, совершив конечное число оборотов, и при $r \rightarrow \infty$ имеет асимптотой полупрямую $\phi=\phi_{\infty}^{* *}$, где

$$
\phi_{\infty}^{* *}=\phi_{0}+\frac{\alpha}{\sqrt{E_{0}^{2}-1}} \int_{r_{0}}^{\infty} d r \sqrt{\frac{\left(r-B Z_{q}\right)}{r\left(r-Z_{q}\right)\left(r+a_{3}^{2}\right)\left(r-r_{2}\right)\left(r-r_{1}\right)}} .
$$

6в. Если $r_{2} \leq Z_{q}<r_{1}$, то траектория движущейся частицы целиком находится в области $r \geq r_{1}$ и ее поведение аналогично разобранному в п. $6 б$.

6г. Если $r_{2}<Z_{q}=r_{1}$, то, как следует из выражения $(13), E_{0}^{2}=(1-B)\left(1+\alpha^{2} / Z_{q}^{2}\right)$ и величина $\alpha^{2}$, помимо условия (19), должна удовлетворять дополнительному неравенству $\alpha^{2}>B Z_{q}^{2} /(1-B)$, обеспечивающему вьполнение условия $E_{0}^{2}>1$.

В этом случае уравнение траектории приобретает качественно новые черты, связанные с тем, что хотя условие неотрицательности правой части равенства (11) и допускает движение частицы во всей области $Z_{q} \leq r<\infty$, подынтегральное выражение (26) имеет особенность вида $\left[r-Z_{q}\right]^{-1}$ в точке $r=Z_{q}$.

Это означает, что при $r=Z_{q}$ интеграл (26) расходится. Поэтому к окружности $r=Z_{q}$ траектория приближается асимптотически, закручиваясь вокруг нее по спирали бесконечное число раз. Так как подынтегральное выражение равенства (26) при $r \rightarrow \infty$ убьвает пропорционально $r^{-2}$, то при $r_{0}>Z_{q}$ и $r \rightarrow \infty$ этот интеграл сходится. Поэтому другая ветвь этой траектории уходит на пространственную бесконечность, совершив конечное число оборотов вокруг тяготеюшего центра, и при $r \rightarrow \infty$ имеет асимптотой полупрямую $\phi=\phi_{\infty}^{* * *}$, где

$$
\phi_{\infty}^{* * *}=\phi_{0}+3 \sqrt{3} B Z_{q} \int_{r_{0}}^{\infty} \frac{d r \sqrt{\left(r-B Z_{q}\right)}}{\left|2 r-3 B Z_{q}\right| \sqrt{r\left(r-Z_{q}\right)\left(r+3 B Z_{q}\right)}} .
$$

Таким образом, при $r_{0}>Z_{q}$ и $d r / d t>0$ массивная частица уходит на бесконечность, а при $d r / d t<0$ она асимптотически стремится к окружности $r=Z_{q}$ и, следовательно, захватывается тяготеюшим центром.

Следует отметить, что наличие кратных корней у правой части выражения (11) является указанием на возможность сушествования круговых орбит. Действительно, подставляя $r=Z_{q}$ в выражения (11), (12), несложно убедиться, что при выполнении условия $E_{0}^{2}=(1-B)\left(1+\alpha^{2} / Z_{q}^{2}\right)>1$ приходим к уравнениям кругового движения $d r / d \phi=d^{2} r / d \phi^{2}=0$. 
6д. Наконец, при $0<r_{2}<r_{1}<Z_{q}$ траектория частицы расположена в области $r \geq Z_{q}$ и ее поведение аналогично поведению траектории, рассмотренной в п. 6б, за исключением того, что точкой поворота траектории будет $r=Z_{q}$.

7. Изучим теперь траектории, которые могут быть реализованы при $E_{0}^{2}>1$ и выполнении условия (20). В этом случае $r_{1}=r_{2}$ :

$$
\begin{aligned}
& r_{2}=\frac{B Z_{q}}{3\left(E_{0}^{2}-1\right)}\left\{\sqrt[3]{1+\frac{9\left(3 E_{0}^{2}-2\right)}{16}\left[27 E_{0}^{4}-36 E_{0}^{2}+8 \sqrt{E_{0}^{2}\left(9 E_{0}^{2}-8\right)^{3}}\right]}-1\right\} \\
& r_{3}=-\frac{B Z_{q}}{3\left(E_{0}^{2}-1\right)}\left\{2 \sqrt[3]{1+\frac{9\left(3 E_{0}^{2}-2\right)}{16}\left[27 E_{0}^{4}-36 E_{0}^{2}+8 \sqrt{E_{0}^{2}\left(9 E_{0}^{2}-8\right)^{3}}\right]}+1\right\}
\end{aligned}
$$

и в зависимости от величины постоянных $E_{0}^{2}, B$ и $Z_{q}$ корни $r_{1}=r_{2}$ могут иметь различное расположение относительно точки $r=Z_{q}$.

7а. Если $Z_{q}<r_{2}=r_{1}$, то условие неотрицательности правой части выражения (11) допускает движение массивной частищы во всей области $Z_{q} \leq r<\infty$. Однако так как в точке $r=r_{1}$ интеграл в выражении (26) расходится, то эта область будет разбита на две подобласти: $Z_{q} \leq r<r_{1}$ и $r_{1}<r<\infty$, в каждой из которых имеется своя траектория. Несложный анализ показывает, что поведение траекторий в этих областях аналогично поведению траекторий, описанных в п. 4.

Так как при $E_{0}^{2}>1$ и выполнении условия (20) корень уравнения (13) $r=r_{2}=r_{1}$ является кратным, то в данном случае оказывается возможным движение массивной частицы и по окружности $r=r_{2}$.

7б. Если $r_{2}=r_{1}=Z_{q}$, то движение массивной частицы будет возможным при $r \geq Z_{q}$. Учитьвая, что в этом случае $r_{3}=-a_{3}^{2}<0$, выражение $(26)$ приведем к виду

$$
\phi=\phi_{0}+\frac{\alpha}{\sqrt{E_{0}^{2}-1}} \int_{r_{0}}^{r} d r \sqrt{\frac{\left(r-B Z_{q}\right)}{r\left(r-Z_{q}\right)^{3}\left(r+a_{3}^{2}\right)}} .
$$

Отсюда непосредственно следует, что один конец траектории массивной частицы будет асимптотически приближаться к окружности $r=Z_{q}$, в то время как другой конец уйдет на пространственную бесконечность. Кроме того, при выполнении указанных выше условий массивная частица может двигаться и по круговой орбите $r=Z_{q}$.

7в. Если $r_{2}=r_{1}<Z_{q}$, то траектория частицы качественно ведет себя так же, как траектория, описанная в п. 6б, за исключением того, что точкой поворота траектории в данном случае будет точка $r=Z_{q}$.

8. Если $E_{0}^{2}>1$ и выполняется условие (21), то корни $r_{1}$ и $r_{2}$ являются комплексно-сопряженными. Вводя обозначение $r_{1,2}=a_{1}+i b_{1}$, в этом случае выражение $(26)$ приведем к виду

$$
\phi=\phi_{0}+\frac{\alpha}{\sqrt{E_{0}^{2}-1}} \int_{r_{0}}^{r} d r \sqrt{\frac{\left(r-B Z_{q}\right)}{r\left(r-Z_{q}\right)\left(r+a_{3}^{2}\right)\left[\left(r-a_{1}\right)^{2}+b_{1}^{2}\right]}} .
$$

Так как условие неотрицательности правой части уравнения (11) в рассматриваемом случае допускает движение во всей области $Z_{q} \leq r<\infty$, то несложно убедиться, что поведение этой траектории аналогично поведению траектории, изученной в п. 5 .

Рассмотрим теперь случай $8 / 9<E_{0}^{2}<1$. 
9. При выполнении условия (22) уравнение (13) имеет три различных положительных вещественных корня: $r_{3}>r_{2}>r_{1}>0$. Интегрируя выражение (11), в этом случае будем иметь

$$
\phi=\phi_{0}+\frac{\alpha}{\sqrt{1-E_{0}^{2}}} \int_{r_{0}}^{r} d r \sqrt{\frac{\left(r-B Z_{q}\right)}{r\left(r-Z_{q}\right)\left(r-r_{1}\right)\left(r-r_{2}\right)\left(r_{3}-r\right)}}
$$

В зависимости от величины постоянных $E_{0}^{2}, \alpha^{2}, B$ и $Z_{q}$ эти корни могут иметь различное расположение относительно точки $r=Z_{q}$. Как показывает детальный анализ, при $1-B \ll 1$ возможны следующие три случая взаимного расположения этих корней относительно точки $r=Z_{q}: Z_{q}<r_{1}<r_{2}<r_{3}, Z_{q}=r_{1}<r_{2}<r_{3}$ и $r_{1}<Z_{q}<r_{2}<r_{3}$.

Рассмотрим эти случаи последовательно.

9а. Если $Z_{q}<r_{1}<r_{2}<r_{3}$, то условие неотрицательности подкоренного выражения в интеграле (27) допускает движение массивной частицы в двух областях пространства: $Z_{q} \leq r \leq r_{1}$ и $r_{2} \leq r \leq r_{3}$.

Так как в этом случае корни функции $(d r / d \phi)^{2}$ являются однократными, то траектория в каждой из указанных областей аналогична траектории, рассмотренной в п. 1а, за исключением того, что точками поворота будут $r=Z_{q}$ и $r=r_{1}$ для траектории, лежашей в первой области, и $r=r_{2}$ и $r=r_{3}$ для траектории, лежашей во второй области.

9б. Если $Z_{q}=r_{1}<r_{2}<r_{3}$, то движение возможно только в области $r_{2} \leq r \leq r_{3}$ или по окружности $r=Z_{q}$. Если частица находится в области $r_{2} \leq r \leq r_{3}$, то выражение (26) принимает вид

$$
\phi=\phi_{0}+\frac{\alpha}{\sqrt{1-E_{0}^{2}}} \int_{r_{0}}^{r} \frac{d r \sqrt{\left(r-B Z_{q}\right)}}{\left(r-Z_{q}\right) \sqrt{r\left(r-r_{2}\right)\left(r_{3}-r\right)}} .
$$

Отсюда непосредственно следует, что на окружностях $r=r_{2}$ и $r=r_{3}$ траектория будет иметь точки поворота и качественно поведение траектории будет аналогичным поведению траектории, описанной в п. 1а.

9в. При $r_{1}<Z_{q}<r_{2}<r_{3}$ движение возможно в области $r_{2} \leq r \leq r_{3}$ и траектория частицы в этой области аналогична траектории п. 1а.

10. Предположим теперь, что $8 / 9<E_{0}^{2}<1$ и выполняется одно из условий $(23)$. В этом случае, полагая в уравнениях (17) $r_{1}=r_{2}$, несложно убедиться, что если $\alpha^{2}=\alpha_{1}^{2}$, то

$$
\begin{aligned}
& r_{1}=r_{2}=\frac{B Z_{q}}{\left(1-E_{0}^{2}\right)}\left\{1-\frac{1}{\sqrt{8}} \sqrt{27 E_{0}^{4}-36 E_{0}^{2}+16+\sqrt{E_{0}^{2}\left(9 E_{0}^{2}-8\right)^{3}}}\right\}>Z_{q}, \\
& r_{3}=\frac{B Z_{q}}{\left(1-E_{0}^{2}\right)}\left\{-1+\frac{1}{\sqrt{2}} \sqrt{27 E_{0}^{4}-36 E_{0}^{2}+16+\sqrt{E_{0}^{2}\left(9 E_{0}^{2}-8\right)^{3}}}\right\}>r_{1} .
\end{aligned}
$$

Если же $\alpha^{2}=\alpha_{2}^{2}$, то

$$
\begin{aligned}
& r_{1}=r_{2}=\frac{B Z_{q}}{\left(1-E_{0}^{2}\right)}\left\{1-\frac{1}{\sqrt{8}} \sqrt{27 E_{0}^{4}-36 E_{0}^{2}+16-\sqrt{E_{0}^{2}\left(9 E_{0}^{2}-8\right)^{3}}}\right\}>Z_{q}, \\
& r_{3}=\frac{B Z_{q}}{\left(1-E_{0}^{2}\right)}\left\{-1+\frac{1}{\sqrt{2}} \sqrt{27 E_{0}^{4}-36 E_{0}^{2}+16-\sqrt{E_{0}^{2}\left(9 E_{0}^{2}-8\right)^{3}}}\right\}>r_{1} .
\end{aligned}
$$


Так как выражение (11) в этих случаях принимает вид

$$
\left(\frac{d r}{d \phi}\right)^{2}=\frac{\left(1-E_{0}^{2}\right) r\left(r-Z_{q}\right)\left(r_{3}-r\right)\left(r-r_{1}\right)^{2}}{\alpha^{2}\left(r-B Z_{q}\right)}
$$

то условие неотрицательности правой части допускает движение массивной частицы только в области $Z_{q} \leq r \leq r_{3}$.

Интегрируя это соотношение, получим

$$
\phi=\phi_{0}+\frac{\alpha}{\sqrt{1-E_{0}^{2}}} \int_{r_{0}}^{r} \frac{d r \sqrt{\left(r-B Z_{q}\right)}}{\left|r-r_{1}\right| \sqrt{r\left(r-Z_{q}\right)\left(r_{3}-r\right)}} .
$$

В области движения $Z_{q} \leq r \leq r_{3}$ подынтегральное выражение имеет особенность вида $\left[r-r_{1}\right]^{-1}$ в точке $r=r_{1}$ и особенности вида $\left[r-Z_{q}\right]^{-1 / 2}$ и $\left[r_{3}-r\right]^{-1 / 2}$ в точках $r=Z_{q}$ и $r=r_{3}$, соответственно. Отсюда непосредственно следует, что область $Z_{q} \leq r \leq r_{3}$ будет разбита на две подобласти $Z_{q} \leq r \leq r_{1}$ и $r_{1} \leq r \leq r_{3}$, в каждой из которых будет находиться своя траектория.

В первой из этих областей точка $r=Z_{q}$ будет точкой поворота траектории, в то время как к окружности $r=r_{1}$ эта траектория будет приближаться асимптотически, совершая бесконечное число оборотов. В подобласти же $r_{1} \leq r \leq r_{3}$ точкой поворота будет точка $r=r_{3}$, а к окружности $r=r_{1}$ траектория будет приближаться асимптотически.

Следует отметить, что в области $Z_{q} \leq r \leq r_{3}$ возможно движение массивной частицы и по круговой орбите радиуса $r=r_{1}$.

11. Пусть $8 / 9<E_{0}^{2}<1$ и выполняется условие $(24)$. В этом случае корни $r_{2}$ и $r_{1}$ уравнения (13) являются комплексно-сопряженными. Обозначая их $r_{1,2}=a \pm i b$, из выражения (11) получим

$$
\left(\frac{d r}{d \phi}\right)^{2}=\frac{\left|E_{0}^{2}-1\right| r\left(r-Z_{q}\right)\left(r_{3}-r\right)\left[(r-a)^{2}+b^{2}\right]}{\alpha^{2}\left(r-B Z_{q}\right)} .
$$

Условие неотрищательности правой части этого выражения допускает движение частицы с указанными выше постоянными $E_{0}^{2}$ и $\alpha^{2}$ только в области $Z_{q}<r<r_{3}$. Траектория массивной частишы в этой области будет поочередно касаться окружностей $r=Z_{q}$ и $r=r_{3}$, обращаясь вокруг тяготеюшего центра.

12. Рассмотрим теперь случай $E_{0}^{2}=8 / 9$. Как следует из выражений (11) и (25), при $\alpha^{2} \neq 3 B^{2} Z_{q}^{2}$ движение массивной частицы будет происходить в области $Z_{q}<r<r_{3}$ и поведение траектории полностью аналогично поведению траектории, рассмотренной в п. 1a.

Если же выполняются условия $E_{0}^{2}=8 / 9$ и $\alpha^{2}=3 B^{2} Z_{q}^{2}$, то движение оказывается возможным в области $Z_{q} \leq r \leq 3 B Z_{q}$ и уравнение траектории принимает вид

$$
\phi=\phi_{0}+\frac{\alpha}{\sqrt{1-E_{0}^{2}}} \int_{r_{0}}^{r} \frac{d r \sqrt{\left(r-B Z_{q}\right)}}{\sqrt{r\left(r-Z_{q}\right)\left(3 B Z_{q}-r\right)^{3}}} .
$$

Анализ подынтегрального выражения показывает, что точка $r=Z_{q}$ является точкой поворота траектории, в то время как к окружности $r=3 B Z_{q}$ траектория приближается асимптотически, совершая бесконечное число оборотов. Так как корень $r=3 B Z_{q}$ 
уравнения (13) имеет кратность, равную трем, то массивная частица может двигаться и по окружности радиуса $r=3 B Z_{q}$.

Заканчивая рассмотрение всех возможных случаев, отметим, что при $E_{0}^{2}<8 / 9$ дискриминант $D$ оказывается положительным при любых значениях $\alpha^{2}$ и, следовательно, уравнение (13) имеет один положительный корень $r=r_{3}$ и два комплексно-сопряженных независимо от величины $\alpha^{2}$. Тогда, поступая аналогично проделанному в п. 11, можно показать, что движение массивной частицы возможно лишь в области $Z_{q}<r<r_{3}$. Качественно поведение траектории в этой области оказывается аналогичным поведению траектории, описанной в п. 1а.

Таким образом, неравенство нулю массы гравитона приводит к тому, что число различных типов траекторий массивных частиц в гравитационном поле статической сферически-симметричной сферы оказывается больше числа типов траекторий в метрике Шварцшильда.

В заключение автор выражает глубокую благодарность академику А.А. Логунову за постановку задачи и полезные обсуждения.

\section{Список литературы}

[1] А.А. Власов, А. А. Логунов. ТМФ. 1989. Т. 78. С. 323.

[2] Ю. М. Лоскутов. ТМФ. 1990. Т. 82. С. 304.

[3] А. А. Логунов. Лекции по теории относительности и гравитации. М.: Наука, 1987. С. 153.

[4] A. А. Логунов. Релятивистская теория гравитации и принцип Маха. Препринт ИФВЭ 95-128, 1995.

Поступила в редакцию 17.I.1997 г.

\section{V.I. Denissov \\ MOTION OF A MASSIVE PARTICLE IN THE VICINITY OF A SINGULAR SPHERE}

Motion of a massive particle in the vicinity of a singularity of a static spherical symmetric metric of the relativistic theory of gravitation is studied. It is shown that the mass value of graviton leads to the appearence of qualitatively new trajectories of motion. 